\title{
A comprehensive study of the $\delta$ Scuti star AN Lyncis
}

\begin{abstract}
A.-Y. Zhou*
National Astronomical Observatories, Chinese Academy of Sciences, Beijing 100012, PR China

Received 25 September 2000 / Accepted 17 January 2002

Abstract. We present new time-series CCD photometry on the $\delta$ Scuti star AN Lyn. Analyses based on all the available data show that the amplitude of the main frequency increased from 1994 to 2000 at a rate of $0.006 \mathrm{mmag} \mathrm{yr}^{-1}$. Considering all the existing amplitudes, the amplitude variations seem sinusoidal since 1980. In both periods of 1980-1983 and 1994-2001 with data available the amplitudes increased linearly with time. The amplitudes in the earlier 1980s were higher and they increased at a higher rate than those in 1994-2001. Amplitude variations of the other frequencies are also present from season to season. The light variation of AN Lyn can be explained currently by amplitude variability plus multiperiodic pulsation. However, no conclusion can be drawn

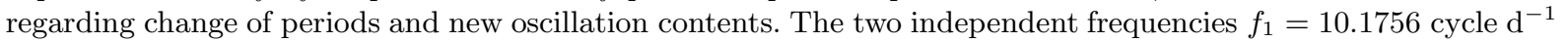
and $f_{2}=18.1310$ cycle $\mathrm{d}^{-1}$ are confirmed and are suggested to be the solution of the light variation of AN Lyn. The mixture of radial and nonradial modes of the two frequencies most likely characterize the pulsation of the star.
\end{abstract}

Key words. stars: variables: $\delta$ Scuti - stars: oscillations - stars: individual: AN Lyn

\section{Introduction}

AN Lyncis $(10.7 \mathrm{~V}, \mathrm{~A} 7 \mathrm{IV}-\mathrm{V})$ is a medium-amplitude multiperiodic $\delta$ Scuti star displaying a relatively simple oscillation spectrum. The principal period of the variable is about 0.09827 with a full $V$ amplitude of $\sim 0^{\mathrm{m}} 18$. AN Lyn was first discovered to be a variable star by Yamasaki et al. (1981). It is a nearly cold and evolved $\delta$ Sct star showing solar metal abundances according to the colour indices and physical parameters derived by Rodríguez et al. (1997a). The earlier works (Agerer et al. 1983; Pensado 1983; Yamasaki et al. 1983; Costa et al. 1984; Poretti et al. 1990) showed that AN Lyn was a monoperiodic $\delta$ Sct pulsator. But the recent Strömgren $u v b y \mathrm{H}_{\beta}$ photoelectric photometry (Rodríguez et al. 1997a, 1997b, hereinafter R97a, R97b) demonstrated that the pulsation of AN Lyn is multiperiodic. Rodríguez et al. (1997b) found three independent frequencies $f_{1}=10.1756, f_{2}=18.1309$

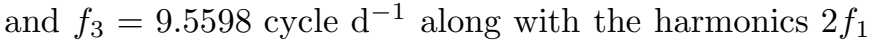
and their interaction terms $f_{1}+f_{2}$ and $2 f_{1}+f_{2}$. Based on the pulsation constant and the analysis of phase shifts between the observed light and colour variations, R97a concluded that the main frequency $\left(f_{1}\right)$ of this star corresponds to the second overtone of radial pulsation. AN Lyn together with V798 Cyg, V1719 Cyg and V974 Oph form a subgroup of the $\delta$ Sct stars exhibiting asymmetric light curves, in which the descending branches are steeper than the ascending ones (Poretti \& Antonello 1988; R97a).

\footnotetext{
* e-mail: aiying@bao.ac.cn
}

However, the peculiarity in the light curves of AN Lyn is dominated by the main frequency and is not due to multiperiodicity as is that of V974 Oph. Rodríguez et al. (1997b) failed to explain the behaviour of its main period from a classic $\mathrm{O}-\mathrm{C}$ analysis. According to these authors, it may be owing to an atypical phase shift between the second and first harmonics (i.e. $2 f_{1}$ and $f_{1}$ ) of the main frequency. Rodríguez et al. (1997b) further confirmed the presence of amplitude variations from one observational season to another for the main frequency as well as for the secondary frequencies $\left(f_{2}\right.$ and $\left.f_{3}\right)$. Nevertheless, they are still not unambiguous about the behaviour of the light variations of AN Lyn.

The aim of this paper is to study the multiperiodic nature and the amplitude variability of AN Lyn based on a comprehensive analysis of the new CCD data and the data available in the literature. Sect. 2 contains a short description of the CCD photometry. The time-dependent behaviour of the star was investigated in Sect. 4. Finally, our main results were summarized and discussed in Sect. 5 .

\section{Data acquisition}

The CCD Johnson $V$ photometry of AN Lyn was carried out with the CCD light-curve survey photometer (Wei et al. 1990) mounted on the 85-cm telescope at the Xinglong Station of the Beijing Astronomical Observatory (BAO) of China. The CCD camera is based on a redsensitive Thomson TH7882 $576 \times 384$ CCD with a readout 
noise of about 10 electrons per pixel. The whole CCD has an imaging size of $13.25 \times 8.83 \mathrm{~mm}^{2}$ which corresponds to a sky field-of-view of $11.5 \times 7 ! 7$. Normally about 10 stars (limited by the current computer programs) were toggled in a CCD frame as a reference. Exposure times ranged from 20 to $60 \mathrm{~s}$ depending on nightly seeing and background contribution. Finally, three comparison stars were selected to produce differential magnitudes for the program star: GSC 02990-00019 $\left(\mathrm{C} 1, \mathrm{RA}=09^{\mathrm{h}} 14^{\mathrm{m}} 34^{\mathrm{s}} .77\right.$, Dec $=42^{\circ} 41^{\prime} 02^{\prime \prime}$ 7, 2000.0, 10.6 V), GSC 02990-00139 (C2, $\mathrm{RA}=09^{\mathrm{h}} 14^{\mathrm{m}} 41^{\mathrm{s}} 41$, Dec $\left.=42^{\circ} 41^{\prime} 11^{\prime \prime} \cdot 3,2000.0,12.8 \mathrm{~V}\right)$ and GSC 02990-001 $\left(\mathrm{C} 3, \mathrm{RA}=09^{\mathrm{h}} 14^{\mathrm{m}} 36.60\right.$, Dec $=$ $\left.42^{\circ} 48^{\prime} 25^{\prime \prime} 7,2000.0,12.2 \mathrm{~V}\right)$.

The CCD photometer system deals with bias and flatfield corrections on-line. The effects of atmospheric and colour extinction have been corrected, even though often negligible. We did not find variability in the light curves of the three comparison stars within the accuracy of our observations. We calculated the magnitude differences of the variable mainly relative to the comparison star $\mathrm{C} 1$. On two nights we took the mean combination as $\mathrm{V}-(\mathrm{C} 1+\mathrm{C} 2+\mathrm{C} 3) / 3$ and on one night we took $\mathrm{V}-\mathrm{C} 2$. A few data points with higher residuals $(>3 \sigma)$ were deleted. $\sigma$ is the nightly standard deviation of the differential magnitudes. Most of the data have been merged into 120-s bins and all normalized to zero. A journal of the observations is given in Table 1. The data set included 21 nights (129 hours) spanning 62 days. There are 2937 measurements in total.

\section{Frequency analysis}

The Fourier frequency analysis was carried out by using PERIOD98 (Breger 1990; Sperl 1998). To judge whether a frequency peak is significant or not we followed the empirical criterion of Breger et al. (1993), that an amplitude signal-to-noise $(S / N)$ ratio larger than four usually corresponds to a peak intrinsic to the target. Using the residuals at the original measurements with all the significant frequencies prewhitened, noise levels at each frequency were computed. Then the confidence levels of the frequencies were estimated according to Scargle (1982). The difference between Strömgren $y$ and Johnson $V$ filters is marginal and negligible, so we analyzed our new $V$ data together with the $y$ data of Rodríguez et al. (1997a, 1997b). The data sets were summarized in Table 2 . They covered a time span of 2246 days.

We found quite a number of peaks spread over the lower frequency region $\left(0-8\right.$ cycle $\left.\mathrm{d}^{-1}\right)$, but the significant peaks were mainly distributed around the integer

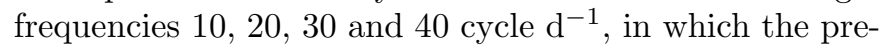
viously detected frequencies $f_{1}, f_{2}, 2 f_{1}, f_{1}+f_{2}$ and $2 f_{1}+f_{2}$ are located. We are aware of the uncertainty of low frequency pulsation because of the motion of images on the CCD chip and possibly the drift of the instruments during the observing seasons. In addition, the effects of arbitrary zero-point adjustments, extinction (atmospheric changes, e.g. variations in sky transparency and scintillation noise)
Table 1. Journal of CCD Johnson $V$ photometry of AN Lyn.

\begin{tabular}{ccc}
\hline $\begin{array}{c}\text { Date } \\
\text { yyyymmdd }\end{array}$ & $\begin{array}{c}\text { HJD(start) } \\
2451000+\end{array}$ & $\begin{array}{c}\text { Measurements } \\
(120-\text { s bins })\end{array}$ \\
\hline 20000208 & 583.01776 & 236 \\
20000209 & 584.32202 & 50 \\
20000210 & 585.32196 & 55 \\
20000211 & 585.99176 & 210 \\
20000212 & 586.97021 & 153 \\
20000217 & 591.99701 & 173 \\
20000218 & 593.25476 & 33 \\
20000219 & 593.96997 & 223 \\
20000220 & 594.96387 & 239 \\
20000221 & 595.95648 & 140 \\
20000222 & 596.96149 & 166 \\
20000223 & 597.95496 & 239 \\
20000224 & 598.95953 & 152 \\
20000225 & 599.96259 & 145 \\
20000301 & 605.01288 & 80 \\
20000328 & 631.99237 & 125 \\
20000329 & 632.98566 & 119 \\
20000330 & 634.02582 & 111 \\
20000404 & 639.00732 & 107 \\
20000409 & 644.01917 & 115 \\
20000410 & 644.99841 & 66 \\
\hline
\end{tabular}

Table 2. Data sets analyzed for AN Lyn.

\begin{tabular}{ccccc}
\hline Year & Nights & Filter & Measurements & Source \\
\hline 1994 & 3 & $y$ & 70 & R97a \\
1995 & 11 & $y$ & 395 & R97a \\
1996 & 16 & $y$ & 531 & R97b \\
2000 & 21 & $V$ & 2937 & present work \\
\hline
\end{tabular}

and similar phenomena not caused by the variable, cannot be separated for frequencies lower than about $5 \mathrm{cy}-$ cle $\mathrm{d}^{-1}$. This low-frequency noise pattern is also visible in the power spectra of R97b. In order to investigate any possible intrinsic stellar variability in low-frequency domain and verify the detection of higher frequencies, an attempt was made to reduce the low-frequency noise in a manner similar to that applied to the $\delta$ Sct star XX Pyx by Handler et al. (2000).

We first re-checked the constancy of the comparison stars in the investigated frequency range. The $(\mathrm{C} 1-\mathrm{C} 2)$ time series from the 11 photometric-quality nights on 8 , 11, 12, 22, 25 February, 28, 29, 30 March and 4, 9, 10 April were thus analysed. We did not find any significant frequencies intrinsic to the comparison stars. For the light curves of the variable, we removed those peaks lower than 8 cycle $\mathrm{d}^{-1}$ with $S / N<4$, through successive prewhitening and least-squares fitting. However, the low-frequency noise is strong and complicated in the $V$ and $y+V$ data 
sets. It cannot be completely filtered. Hence, we stopped searching for pulsation frequencies in the low-frequency region. We show the reduced residual spectra in Fig. 1, where the main frequency $f_{1}=10.1756$ cycle $\mathrm{d}^{-1}$ was also removed from the data.

Besides the existing aliases in the power spectra of the combined data, there are two strong peaks around the main frequency at 10.1726 and 10.1836 cycle $\mathrm{d}^{-1}$, which differ from $f_{1}=10.1757$ cycle $\mathrm{d}^{-1}$ by 0.0031 and 0.0079 cycle $\mathrm{d}^{-1}$, respectively. However, they are two spurious terms introduced by an amplitude modulation of $f_{1}$. Owing to this modulation, the peak at $f_{1}$ is larger than the theoretical one resulting from a signal having a constant amplitude. As a consequence, the residual signal after removing $f_{1}$ generates two spurious peaks at each side and very close to the main peak. On the other hand, the amplitude modulation also caused higher noise in the region around $f_{1}$. However, these spurious terms and higher noise are not present in the spectra of the present data alone (see the top panel of Fig. 1). Particular caution must be taken if the data are to be used to search for pulsation frequencies lower than $f_{1}$. It is seen also from Fig. 1 that both the peaks at $f_{2}=18.1310$ cycle $\mathrm{d}^{-1}$ and $f_{2}-1$, an alias of $f_{2}$, have similar heights. The reason to prefer $f_{2}$ to $f_{2}-1$ is based on three aspects: the period-search programs (PERIOD98 and MFA) pick $f_{2}$ up rather than $f_{2}-1$; the detection of $f_{1}+f_{2}$ and $2 f_{1}+f_{2}$; for the $y$ data of R97a and R97b, their results and our reanalysis using different routines consistently suggest $f_{2}$.

As a result, we failed to detect any new frequencies of oscillation and to verify $f_{3}=9.5598$ cycle $\mathrm{d}^{-1}$ of R97b. The reanalysis of the $y$ data alone does not confirm the term $f_{3}$ either (see the mid panel of Fig. 1). Hence this frequency is retained as uncertain. The results for the two individual data sets corresponding to filters $V$ and $y$ were given in Table 3, while those for their combination in Table 4. Except for the last term with a degree of confidence of about $90 \%$, all other terms are reliable. Finally, we consider the two frequencies $f_{1}$ and $f_{2}$ to be intrinsic to AN Lyn and we select the five frequency contents $f_{1}=10.1757, f_{2}=18.1310,2 f_{1}=20.3514$, $f_{1}+f_{2}=28.3067$ and $2 f_{1}+f_{2}=38.4824$ cycle $\mathrm{d}^{-1}$ to represent the pulsational behaviour of AN Lyn. The 5 -sinusoid least-squares fitting resulted in a standard deviation $\sigma=0$. 0149 for the combined data, which shows a slight insufficiency of fitting as compared with the observational accuracy. The poor fit largely resulted from the higher noise in the low frequency domain as already mentioned.

The results using PERIOD98 were checked against another similar multi-frequency analysis program MFA (Hao 1991; Liu 1995). The errors on the fitted frequencies estimated from nonlinear fitting are in the range from 0.0001 cycle $\mathrm{d}^{-1}$ for $f_{1}$ to 0.0043 cycle $\mathrm{d}^{-1}$ for $2 f_{1}+f_{2}$, while the errors on amplitudes, about 0.0002 , are independent of frequencies. The errors are in accordance with the estimations following the formulae of Montgomery \& O'Donoghue (1999). The CCD differential light curves

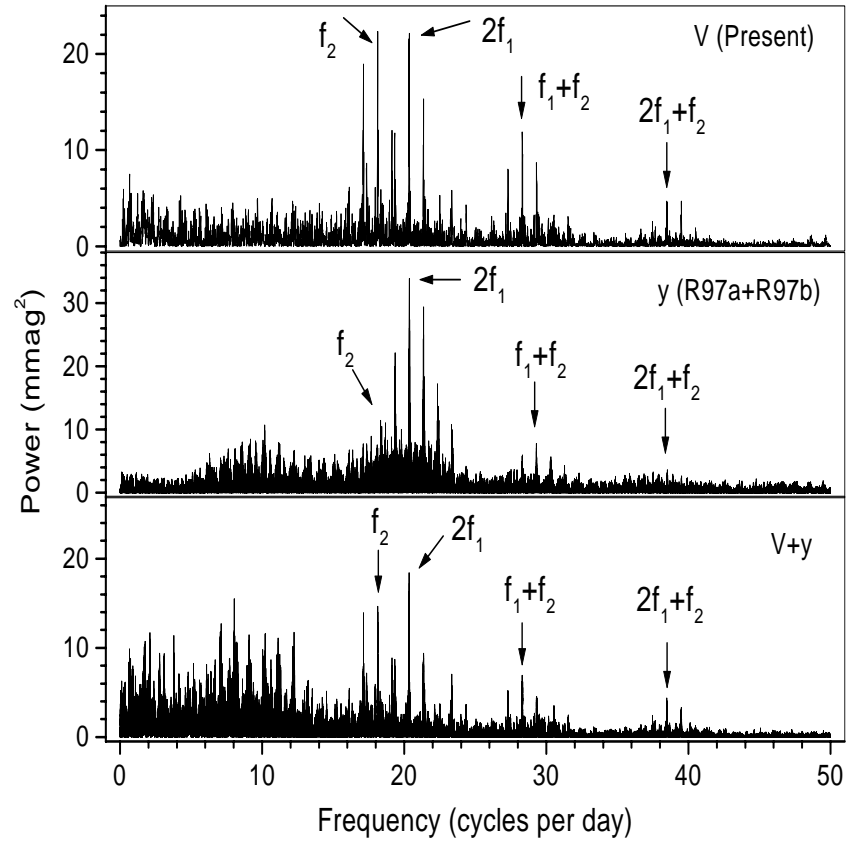

Fig. 1. Low-frequency-denoised spectra for the two sets of data $(V$ and $y)$ and their bins $(V+y)$. Higher noise level still exists in the binned data due to amplitude variability of the main frequency at 10.1757 cycle $\mathrm{d}^{-1}$.

Table 3. Trial frequency $\left(\right.$ cycle $\mathrm{d}^{-1}$ ) solutions of AN Lyn for the new CCD data and the data of Rodríguez et al. (1997a, 1997b). Amplitudes and fitting errors are given in millimagnitudes.

\begin{tabular}{rlrlr}
\hline \multicolumn{1}{c}{ Sets } & \multicolumn{2}{c}{ Present } & \multicolumn{2}{c}{ R97a+R97b } \\
Terms & Freq. & Ampl. & \multicolumn{1}{l}{ Freq. } & Ampl. \\
\hline$f_{1}$ & 10.1756 & 82.0 & 10.1757 & 69.0 \\
$2 f_{1}$ & 20.3518 & 5.1 & 20.3513 & 6.3 \\
$f_{2}$ & 18.1315 & 5.3 & 18.1311 & 3.6 \\
$f_{3}$ & - & - & 8.6052 & 3.9 \\
$f_{1}+f_{2}$ & 28.3063 & 3.6 & 28.3045 & 2.9 \\
$2 f_{1}+f_{2}$ & 38.4833 & 2.1 & 38.4824 & 1.6 \\
\hline$\sigma$ & \multicolumn{3}{c}{12.1} & \multicolumn{3}{c}{8.9} \\
\hline
\end{tabular}

of AN Lyn along with the fit of the 5-term solution are presented in Fig. 2. Our calculated light curves fit the observed ones quite well. Some points where the observed light curve deviates from the calculated one are due largely to photometric errors or instrumental effects. We have noted the possible systematic deviations between observations and fit at times such as 18, 19, 20 and 22 February 2000 in Fig. 2. When we discarded these measurements and analysed the rest, the Fourier solutions did not change.

\section{Time-dependent behaviours of AN Lyn}

For signals with consistent periodic fluctuation, such as the pulsation of $\delta$ Sct stars, the weighted wavelet Ztransform (WWZ) (Foster 1996) is an ideal tool to track 

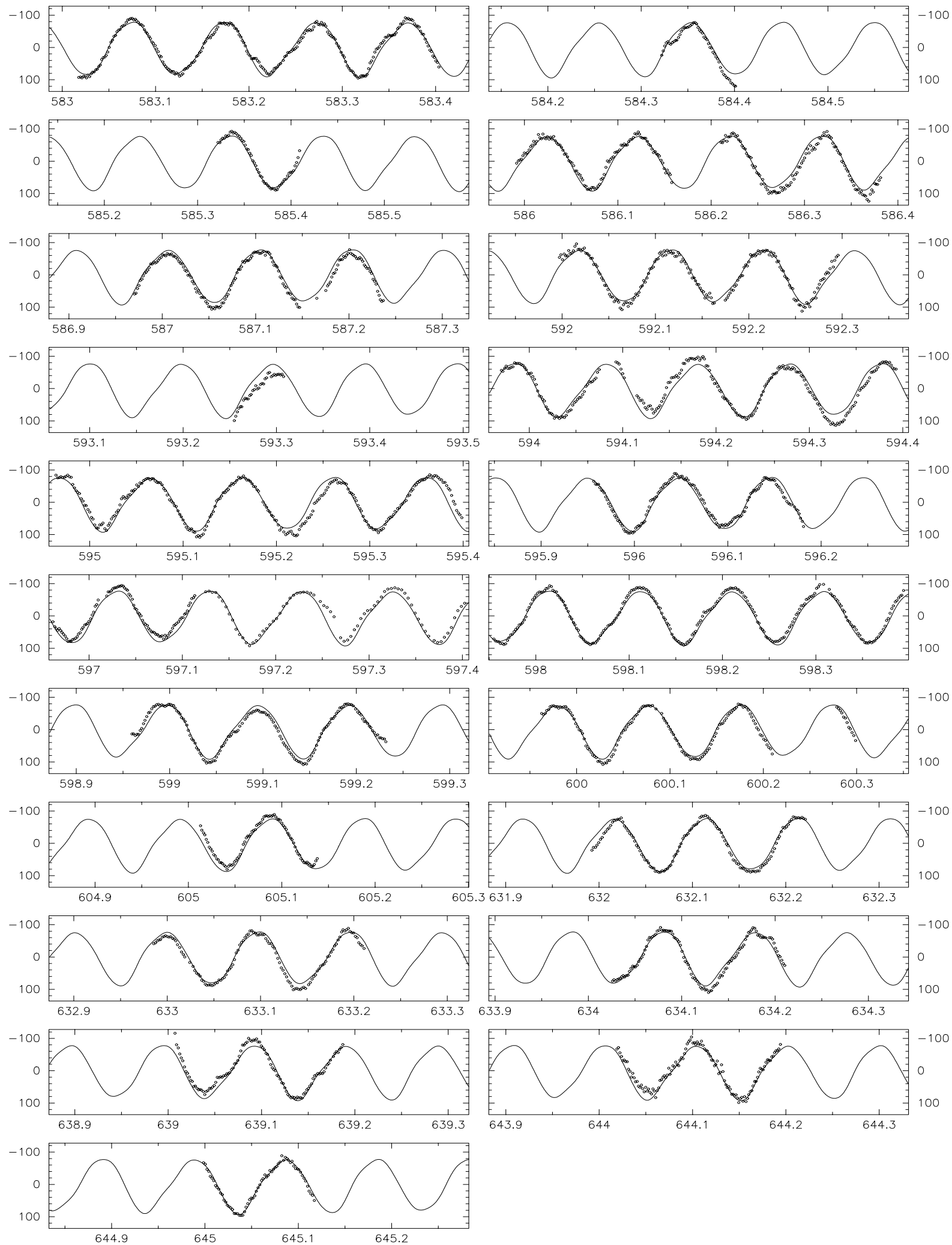

Fig. 2. CCD $V$ differential light curves (circles) of AN Lyn together with the 5-frequency fitted sinusoids represented by solid lines. Abscissa is the time in HJD 2451000+ days, ordinate in milli-magnitudes.

the time evolution of the parameters such as period and amplitude. Because the wavelet transform locates information in both frequency and time domains, one may focus on one of them for different information of interest.
First, we focus on the dependence of the intensity of a signal on time, that is, the wavelet amplitude variation of the main frequency of AN Lyn. Then, we pay attention to the dependence of the intensity of the signal on frequency, 
Table 4. Frequency contents of AN Lyn based on all the data in Table 2. Epoch in HJD 2451583.0+ days.

\begin{tabular}{llrcr}
\hline & $\begin{array}{c}\text { Freq. } \\
\left(\text { cycle d }^{-1}\right)\end{array}$ & $\begin{array}{r}\text { Ampl. } \\
(\mathrm{mmag})\end{array}$ & $\begin{array}{r}\text { Phase, Epoch } \\
(0-1), \text { day }\end{array}$ & $S / N$ \\
\hline$f_{1}$ & $10.1757 \pm .0001$ & $73.6 \pm .2$ & $.8992, .0411$ & 57.1 \\
$2 f_{1}$ & 20.3514 & $4.7 \pm .2$ & $.6399, .0135$ & 7.9 \\
$f_{2}$ & $18.1310 \pm .0013$ & $4.3 \pm .2$ & $.1322, .0612$ & 6.6 \\
$f_{1}+f_{2}$ & 28.3067 & $2.7 \pm .2$ & $.6922, .0442$ & 5.6 \\
$2 f_{1}+f_{2}$ & 38.4824 & $1.3 \pm .2$ & $.0288, .0645$ & 3.6 \\
\hline
\end{tabular}

that is, the variation of a frequency. Based on our new CCD data and the photoelectric data used in Rodríguez et al. (1997a, 1997b), the time-evolution behaviours of the light curves of AN Lyn were investigated by applying WWZ. We have detected the time-dependence of the main frequency's amplitude, but variations of periods are still inconclusive.

\subsection{Amplitude variations}

The new CCD data provide an excellent opportunity to examine the phenomenon of amplitude variability of AN Lyn claimed by R97b. We set frequency in the range of 10.165 to 10.185 around the main frequency 10.1756 cycle $\mathrm{d}^{-1}$. This means a very small frequency variation up to 0.020 cycle $\mathrm{d}^{-1}$ was allowed. In other words, the main frequency was constant in a 0.02 cycle $\mathrm{d}^{-1}$ level, which is far from possible changes. The time varied from HJD 2449400 to 2451640 days crossing the years from 1994 to 2000. Consequently, the WWZ output the amplitude information of the main frequency $f_{1}=10.1756 \pm$ 0.0002 cycle $\mathrm{d}^{-1}$ over the time duration located. The wavelet analysis results are plotted in Fig. 3, where the Fourier amplitudes are also shown for comparison. By fixing the main frequency $f_{1}=10.1756$ cycle $\mathrm{d}^{-1}$ and allowing amplitude and phase variations, we did singlefrequency least-squares sinusoid fit to different data sets to determine the amplitudes. Then we obtained 0.0673 , 0. $0675,0.0718$ and $0 .{ }^{\mathrm{m}} 0807$ for the years 1994,1995 , 1996 and 2000, respectively. The amplitudes from the wavelet analysis are in well agreement with those derived by Fourier analysis. The values for the former three years are consistent with those given by R97b. From the results we are convinced that the amplitude of the main frequency of AN Lyn was increasing from 1994 to 2000. An attempt at linear-fitting to the variations results in

$\Delta V=-0.25 \pm 0.02+(6.37 \pm 0.39) \times 10^{-6} t$

with $\sigma=0{ }^{\mathrm{m}} 00165$, which indicates a mean increasing rate of about 6.37 per million years or six thousandth millimagnitudes per year. Here and below we define $t=$ HJD 2400000.0 days. In the same way, by fixing the five frequency terms $f_{1}=10.1756,2 f_{1}=20.3512, f_{2}=18.1310$, $f_{1}+f_{2}=28.3066$ and $2 f_{1}+f_{2}=38.4822$ cycle $\mathrm{d}^{-1}$ we found their amplitude variations by multifrequency

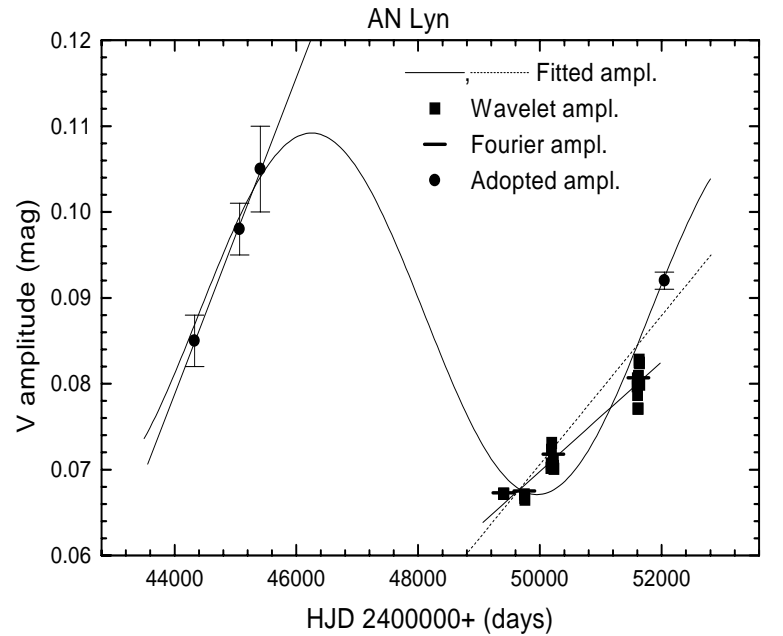

Fig. 3. Time evolution of the $V(y)$ amplitude of the main frequency of AN Lyn. Three individual linear fits to the Fourier amplitudes in 1980-1983 and in 1994-2001 (dotted line) and to the wavelet amplitudes in 1994-2000 are shown. The sinusoid is a fit of all the Fourier (adopted) amplitudes. The Fourier amplitudes notated as bars having errors of $\sim 00^{\mathrm{m}} 001$ are derived presently or re-derived from existing time-series data.

Table 5. Time-dependence of the $V$ amplitudes (in mmag) of the detected pulsation frequencies of AN Lyn determined by multifrequency least-squares fitting. $\sigma$ is the fitting errors.

\begin{tabular}{lrrcc}
\hline Frequency & $\left(\right.$ cycle d $\left.^{-1}\right)$ & 1995 & 1996 & 2000 \\
\hline$f_{1}$ & 10.1756 & 67.3 & 71.9 & 80.7 \\
$2 f_{1}$ & 20.3512 & 5.6 & 7.1 & 4.8 \\
$f_{2}$ & 18.1310 & 3.8 & 3.0 & 4.8 \\
$f_{1}+f_{2}$ & 28.3066 & 2.6 & 2.9 & 3.3 \\
$2 f_{1}+f_{2}$ & 38.4822 & 0.9 & 1.7 & 1.9 \\
\hline$\sigma$ & & 10.3 & 8.1 & 13.6 \\
\hline
\end{tabular}

least-squares sine-wave fitting. The standard deviations of the residuals of fitting $(\sigma)$ for each data set conform to the observational errors. They are given in Table 5. It is known that the Fourier amplitudes are averaged over a period of time and they are obtained severally from different data sets on the assumption that the frequency is constant in the observing duration. However the wavelet amplitudes are a time-dependent series resolved from a single combined data set.

When the amplitudes in the early 1980s and the latest value of $0 .{ }^{\mathrm{m}} 092 \pm 0.001$ for 2001 (Lacluyzé et al. 2001) are considered, the variations of the amplitude become complicated and quite interesting. According to Yamasaki et al. (1983), Agerer et al. (1983) and Rodríguez et al. (1997b), we estimated the following amplitude values $0.085 \pm 0.003,0.098 \pm 0.003$ and $0 .{ }^{\mathrm{m}} 105 \pm 0.005$ for the main frequency for the years 1980, 1982 and 1983, respectively. We used the "semi-amplitudes" throughout the paper. We disregarded the value of $0{ }^{\mathrm{m}} 1114 \pm 0.0031$ given by Costa et al. (1984) for 1982 because it is somewhat larger 
than that of Yamasaki et al. (1983). We tried a linear fitting to the amplitudes in the 1980s and we got

$\Delta V=-0.7325 \pm 0.0314+(1 . \mathrm{m} 84 \pm 0.07) \times 10^{-5} t$

with $\sigma=0{ }^{\mathrm{m}} 0005$. For the amplitudes from 1994 to 2001, our linear-fitting results are

$\Delta V=-0{ }^{\mathrm{m}} 3634 \pm 0.0732+\left(8^{\mathrm{m}} \cdot 68 \pm 1.45\right) \times 10^{-6} t$

with $\sigma=0$. 0034 (see the dotted line in Fig. 3). The earlier amplitudes in 1980-1983 seemed to increase with a larger rate than those in 1994-2001. If we take all the amplitude values into account together, a sinusoid fit can be attempted:

$\Delta V=0 .{ }^{\mathrm{m}} 0881+0.02105 \sin (2 \pi \times 0.000135 t+0.0392)$

which indicates a possible cyclic amplitude variation with

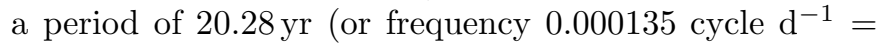
0.0493 cycle $\mathrm{yr}^{-1}$ ). This means the main amplitude has a cyclic variation of about 5 cycles in a century. We show the results in Fig. 3. The error bars for the Fourier amplitudes derived in this work of $\sim 0.001$ are not displayed. The adopted amplitudes with error bars are the estimated values from the literature. Compared to the recent errors of amplitudes $\left(\sim 0^{\mathrm{m}} 001\right)$, the earlier amplitudes have larger errors $\left(\sim 0 \cdot 003-0 \cdot{ }^{\mathrm{m}} 005\right)$. The reality of the possible periodic amplitude variations is very interesting and needs confirmation. Further data will be helpful to examine the pattern of amplitude variations presented here and would hopefully lead to an understanding of the reason causing these cyclic changes.

\subsection{Period variations}

In order to see the temporal-dependence of the main frequency $f_{1}$ during the years with available data, we applied the single-frequency Fourier technique to different data sets using the residuals after prewhitening $f_{2}=$ 18.1310 cycle $\mathrm{d}^{-1}$. We found the values of 10.175577 , 10.175627 and 10.175550 cycle $^{-1}$ for 1995,1996 and 2000 respectively. Concerning the estimated errors in Table 4, the results do not suggest variability of the main frequency. Furthermore, we display an updated O-C diagram in Fig. 4 by appending 53 times of maximum light determined from the present light curves to the existing maxima in R97b. The $\mathrm{O}-\mathrm{C}$ residuals and elapsed cycles $(E)$ are based on the ephemeris: $\mathrm{HJD}_{\max }=2449398.7492+$ $0.09827315 \times E$ (R97b). It is hard to explain the morphology of the $\mathrm{O}-\mathrm{C}$ residuals. Therefore, the variability of the main period of AN Lyn remains open.

\section{Conclusions and discussion}

By making use of the new CCD measurements and the data in the literature, we have investigated the temporaldependent behaviours of the light variations of the $\delta$ Scuti star AN Lyn on a time base of six years. AN Lyn shows evident amplitude variations between 1994 and 2000. Fourier

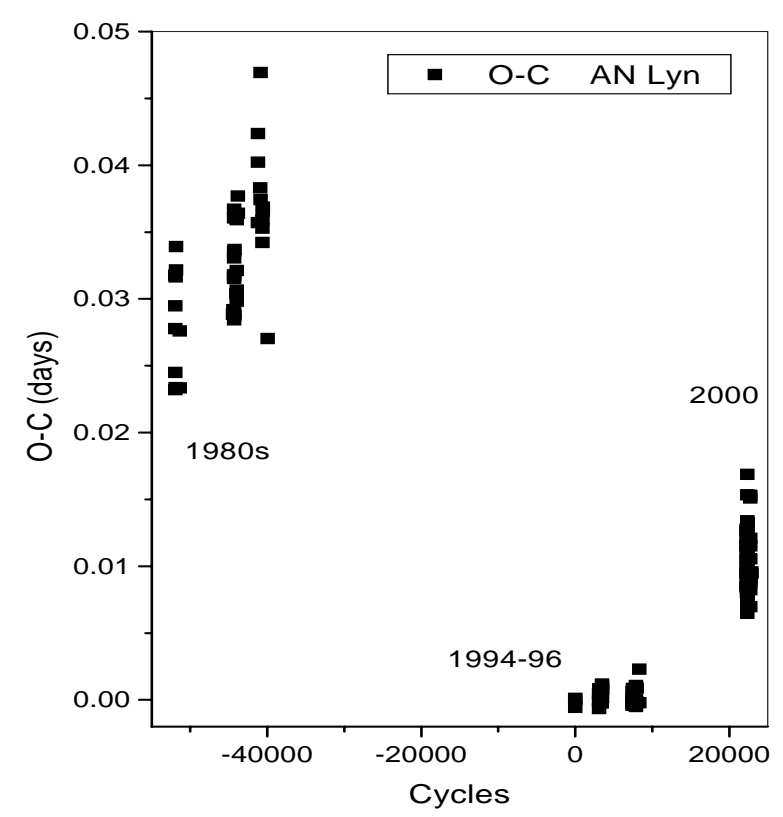

Fig. 4. O-C diagram for AN Lyn, based on 131 times of maximum light up to date.

analysis by fixing the main frequency allowing amplitude variation as well as the wavelet analysis consistently shows that the amplitude of the main frequency $f_{1}$ of AN Lyn was increasing during the years from 1994 to 2000 at a rate of six thousandth milli-magnitudes per year. The amplitude variations of other frequencies are also present from season to season. The application of wavelet transform to the time-series data open us a new way to track the time evolution of any periodic changes such as the amplitudes and frequencies of oscillations in variables.

Furthermore, taking the amplitudes in the 1980s and the most recent value for 2001 into consideration, variations in amplitude of the main frequency $f_{1}$ appear cyclic. We show the evolution in amplitude of $f_{1}$ and our possible fittings in Fig. 3. The sinusoid fit predicts a periodic amplitude variation of about half a cycle per ten years (0.0493 cycle $\mathrm{yr}^{-1}$ ) or a period of $\sim 20 \mathrm{yr}$. Individual linear fit to two parts of the amplitude shows that the variations in the 1980s are steeper than in 1994-2001, which means a larger increasing rate in that time. The amplitude variations are quite interesting. Amplitude variability in $\delta$ Sct stars is common, such as is observed in $\theta^{2}$ Tau (Li et al. 1997), 28 And (Rodríguez et al. 1998), DQ Cep (Li \& Fang 1999) and 4 CVn (Breger 2000). If the cyclic amplitude is real, a similar cyclic variation would be present in the $\mathrm{O}-\mathrm{C}$ diagrams with the same time-scale as for the amplitude modulation. The fact of this potential match will be an argument that AN Lyn is a binary. It is not surprising that the cyclic variations are the results of the motion of the pulsating star (AN Lyn itself) in a binary system. In this regard, the main frequency should show observable changes on this time scale. In turn, a cyclic changing main period together with amplitude modulation could cause a higher noise level in the lower amplitude spectrum as 
seen in our analyses. Concerning the low-frequency noise, as shown in Fig. 1 and de-noised following the method used for XX Pyx by Handler et al. (2000), it is possibly caused by intrinsic low frequencies or binarity as shown by Arentoft et al. (2001a). We have noted a similar sinusoidal amplitude variation appearing in the $\delta$ Sct star V1162 Ori (Arentoft et al. 2001b), for which binarity (Arentoft $\&$ Sterken 2000) and multiperiodicity were proposed and well discussed. Last, however, larger errors for the earlier values should be noted before giving any convincing explanations. Confirmation and any explanation for the present of the interesting amplitude changes should be a focus of future works.

Concerning the current detection of pulsation frequencies based on the present CCD data and the photoelectric data of Rodríguez et al. (1997a, 1997b), the peculiar light variations of AN Lyn are explained by amplitude variability. AN Lyn is another example with a high degree of amplitude variability. However, the origin of the amplitude variability in $\delta$ Scuti stars is presently not understood (Breger 2000). The pulsation of AN Lyn can be represented by the two independent frequencies

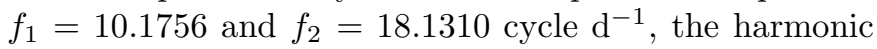
$2 f_{1}=20.3512$ cycle $^{-1}$ and the two cross-coupling terms $f_{1}+f_{2}=28.3066$ and $2 f_{1}+f_{2}=38.4822$ cycle $^{-1}$. The term $f_{3}$ obtained by R97b was not verified in the present analyses.

The radial pulsation mode of $\delta$ Sct stars can be estimated by means of the absolute bolometric magnitude, the pulsation constant and references to the existing theoretic models. Unfortunately, no HIPPARCOS parallax is available for AN Lyn, so we assumed the calibrated Strömgren photometric results $M_{\mathrm{bol}}=1{ }^{\mathrm{m}}$ 0 $(\mathrm{R} 97 \mathrm{a})$. In terms of the theoretical period-luminosity-effective temperature relations for the four lowest radial modes $(\mathrm{F}, 1 \mathrm{O}$, $2 \mathrm{O}$ and $3 \mathrm{O}$ ) of Tsvetkov (1985), $f_{1}$ can be identified to be the second overtone radial mode $(2 \mathrm{O})$, with no identification for $f_{2}$. According to the empirical period-luminositycolour (P-L-C) relations of Stellingwerf (1979) and López de Coca et al. (1990) and adopting the mean values of $M_{\mathrm{bol}}=1{ }^{\mathrm{m}} 0$ and $T_{\text {eff }}=7270 \mathrm{~K}$ from R97a, $f_{1}$ is identified as a $2 \mathrm{O}$ or $3 \mathrm{O}$ mode. The relations give frequency ratios $0.77,0.81,0.68$ and 0.83 for $\mathrm{F} / 1 \mathrm{O}, 1 \mathrm{O} / 2 \mathrm{O}, 1 \mathrm{O} / 3 \mathrm{O}$ and $2 \mathrm{O} / 3 \mathrm{O}$, respectively. However, the observed frequency ratio $f_{1} / f_{2}=0.56$ or $f_{1} /\left(f_{2}-1\right)=0.59$, taking the alias into account, has no counterpart in the expected frequency ratios or their reciprocals. Furthermore, if the P-L relations for high-amplitude $\delta$ Sct stars (or SX Phoenicis stars) derived using the HIPPARCOS results (e.g. Antonello \& Mantegazza 1997; McNamara 1997, 2000; Petersen \& Høg 1998; Petersen 2000) or the P-L-C-metallicity relations valid for the full $\delta$ Sct instability strip derived from stellar evolution and pulsating modeling (Petersen 2000) are considered, a radial fundamental mode at $\sim 6.69$ cycle $^{-1}$ was predicted. Then the first and third radial overtones

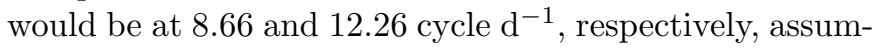
ing $f_{1}$ to be $2 \mathrm{O}$ and the period ratio $P_{1} / P_{0}=0.772$, a generally accepted value for Pop. I metal-strong variables
( $Z \sim 0.01-0.02)$ (McNamara 2000). Obviously the results ruled out the possibility of a radial low overtone for $f_{2}$.

On the other hand, we calculated the pulsation constants $Q_{1}=0.021$ and $Q_{2}=0.012$ for the two frequencies $f_{1}$ and $f_{2}$ using the physical parameters derived in R97a and the formula of Petersen \& Jørgensen (1972). $Q_{1}=0.021$ matched the mean pulsation constant of the $2 \mathrm{O}$ mode $\left(\bar{Q}_{\mathrm{F}}=0.033, \bar{Q}_{1 \mathrm{O}}=0.025, \bar{Q}_{2 \mathrm{O}}=0.020\right.$, $\left.\bar{Q}_{30}=0.017\right)$, while the very low value of $Q_{2}=0.012$ may be due to nonradial oscillations in a high overtone (Tsvetkov 1985). In terms of the 2.0M43 model of Fitch (1981), $f_{1}$ can be identified as the radial $2 \mathrm{O}$ mode, while $f_{2}$ as a nonradial $p_{6}$ mode of $l=1$ or 2 . We noted the deviation in identifications caused by the uncertainty of $Q$ values of $\sim 18 \%$ (or of order 0.005 ) calculated from uvby photometry (Breger et al. 1999; Lampens et al. 1999).

We also derived the radius $R=3.54 \pm 0.4 R_{\odot}$, then the mean density $\rho=0.045 \pm 0.003 \rho_{\odot}\left(M=2.0 M_{\odot}\right)$ assuming R97a's parameters. Referring to the theoretical frequencies as well as frequency ratios of the radial oscillation modes of the $2.2 M_{\odot}$ model $(Z=0.02)$ of Viskum et al. (1998), this density of AN Lyn does not suggest a radial mode at the value of $f_{2}$. Moreover, $f_{2}$ is neither present in the $2.0 M_{\odot}(Z=0.02)$ model with $l=0-2$ of Templeton et al. (1997) nor in the models of Breger et al. (1999) for the well-studied $\delta$ Sct star FG Virginis. At least, the degree $l$ for $f_{2}$ is nonzero. Compared further with the most recent best-fit model for FG $\operatorname{Vir}\left(Z=0.03, M=1.9 M_{\odot}\right)$, Fig. 10 of Templeton et al. (2001), $f_{2}(209.85 \mu \mathrm{Hz})$ can be referred to a nonradial mode of $l=3$ for a non-rotating case or $l=2$ or 3 for a model with first-order rotational splittings of $v \sin i=50 \mathrm{~km} \mathrm{~s}^{-1}$. Consequently, the main pulsation $\left(f_{1}\right)$ of AN Lyn seems to be the $2 \mathrm{O}$ mode, is in agreement with R97a's identification. The oscillation of $f_{2}$ is most likely to be a nonradial mode of $l \geq 2$. A photometric confirmation for the mode of $f_{2}$ is necessary. Thus the nature of mixed radial and nonradial pulsation in the light variations of AN Lyn is established.

As seen, the quality of the CCD data is not high. This seems to be one of the sources causing the noise peaks in the Fourier spectra. Nevertheless, this has no effect on the results of amplitude change. We understand that the analysis based on all the available data was strongly contaminated by the intrinsic amplitude modulations. This prevents us from revealing any other pulsation frequencies on a binned data set of longer time-base. Moreover, the lowfrequency noise is very strong and complicated. Although a particular effort was made to reduce the red noise, it seems not treatable. Future high precision multi-site observations overcoming low-frequency noise and spectral leakage phenomenon are required. The linear increment behaviour of the amplitude of the main frequency can also be examined with additional data.

Acknowledgements. The author is very grateful to Dr. Eloy Rodríguez at IAA (Spain) for supplying his time-series data, and to the anonymous referee for constructive comments which helped to improve the manuscript. This work was supported by the Natural Science Foundation of China. 


\section{References}

Agerer, F., Fernandes, M., \& Frank, P. 1983, IBVS, 2370

Antonello, E., \& Mantegazza, L. 1997, A\&A, 327, 240

Arentoft, T., \& Sterken, C. 2000, A\&A, 354, 589

Arentoft, T., Sterken, C., \& Handler, G. 2001a, MNRAS, 326, 192

Arentoft, T., Sterken, C., Handler, G., et al. 2001b, A\&A, 374, 1056

Breger, M. 1990, Comm. in Asteroseismology 20, 1 (Vienna: Austrian Academy of Sciences)

Breger, M. 2000, MNRAS, 313, 129

Breger, M., Stich, J., Garrido, R., et al. 1993, A\&A, 281, 90

Breger, M., Pamyatnykh, A. A., Pikall, H., \& Garrido, R. 1999, A\&A, 341, 151

Costa, V., Garrido, R., López de Coca, P., et al. 1984, A\&AS, 57,233

Fitch, W. S. 1981, ApJ, 249, 218

Foster, G. 1996, AJ, 112, 1709

Handler, G., Arentoft, T., Shobbrook, R. R., et al. 2000, MNRAS, 318, 511

Hao, J.-X. 1991, Publ. Beijing Astron. Obs., 18, 35

Lampens, P., Van Camp, M., \& Sinachopulos, D. 1999, Delta Scuti Star Newsletter, 13, 10 (Univ. of Vienna)

Lacluyzé, A., Smith, H. A., Clark, A. R., et al. 2001, IBVS, 5180

Li, Z.-P., Zhou, A.-Y., \& Yang, D.-W. 1997, PASP, 109, 217

Li, Z.-P., \& Fang, M.-J. 1999, A\&AS, 136, 515

Liu, Z.-L. 1995, A\&AS, 113, 477

López de Coca, P., Rolland, A., Rodríguez, E., \& Garrido, R. 1990, A\&AS, 83, 51

McNamara, D. H. 1997, PASP, 109, 1221

McNamara, D. H. 2000, in Delta Scuti and Related Stars, ed. M. Breger, \& M. H. Montgomery, ASP Conf. Ser., 210, 373
Montgomery, M. H., \& O'Donoghue, D. 1999, Delta Scuti Star Newletter, 13, 28 (Univ. of Vienna)

Pensado, J. 1983, Bol. Astron. Obs. Madrid, vol. 11, No. 2, 3

Petersen, J. O. 2000, in Delta Scuti and Related Stars, ed. M. Breger, \& M. H. Montgomery, ASP Conf. Ser., 210, 474

Petersen, J. O., \& Høg, E. 1998, A\&A, 331, 989

Petersen, J. O., \& Jørgensen, H. E. 1972, A\&A, 17, 367

Poretti, E., \& Antonello, E. 1988, A\&A, 199, 191

Poretti, E., Antonello, E., \& Le Borgne, J. F. 1990, A\&A, 228, 350

Rodríguez, E., González-Bedolla, S. F., Rolland, A., et al. 1997a, A\&A, 324, 959 (R97a)

Rodríguez, E., González-Bedolla, S. F., Rolland, A., et al. 1997b, A\&A, 328, 235 (R97b)

Rodríguez, E., Rolland, A., López-González, M. J., \& Costa, V. 1998, A\&A, 338, 905

Scargle, J. 1982, ApJ, 263, 835.

Sperl, M. 1998, Comm. in Asteroseismology, 111, 1 (Vienna: Austrian Academy of Sciences)

Stellingwerf, R. F. 1979, ApJ, 227, 935

Templeton, M., Basu, S., Demarque, P. 2001, ApJ, submitted [astro-ph/0108458]

Templeton, M. R., McNamara, B. J., Guzik, J. A., et al. 1997, AJ, 114, 1592

Tsvetkov, T. G. 1985, Ap\&SS, 117, 137

Viskum, M., Kjeldsen, H., Bedding, T. R., et al. 1998, A\&A, 335,549

Wei, M.-Z., Chen, J.-S., \& Jiang, Z.-J. 1990, PASP, 102, 698

Yamasaki, A., Okazaki, A., \& Kitamura, M. 1981, PASP, 93, 77

Yamasaki, A., González-Bedolla, S. F., Peniche, R., \& Peña, J. H. 1983, PASP, 95, 447 